\title{
Experimental spatial rogue patterns in an optical feedback system
}

\author{
V. Odent, M. Taki, and E. Louvergneaux \\ Université Lille1, Laboratoire de Physique des Lasers, Atomes et Molécules, CNRS UMR 8523, \\ 59655 Villeneuve d'Ascq Cedex, France
}

Received: 30 September 2010 - Revised: 22 November 2010 - Accepted: 23 November 2010 - Published: 22 December 2010

\begin{abstract}
We study pattern formation in an optical system composed of a Kerr medium subjected to optical feedback but in a regime very far from the modulational instability threshold. In this highly nonlinear regime, the dynamics is turbulent and the associated one-dimensional patterns depict rare and intense localized optical peaks. We analyse numerically and experimentally the statistics and features of these intense optical peaks and show that their probability density functions (PDF) have a long tail indicating the occurrence of rogue events.
\end{abstract}

\section{Introduction}

Since the experimental evidence of temporal optical rogue waves in a fibre system by Solli and coworkers (Solli et al., 2007), rogue wave research activity has received a considerable attention in optics in general (Solli et al., 2008; Dudley et al., 2008; Hammani et al., 2008; Mussot et al., 2009; Montina et al., 2009). Such rare and intense events are originally observed, e.g., in open ocean in form of rogue or freak waves (Kharif and Pelinovsky, 2003; Broad, 2006; Fedele, 2008) and more recently in, for example, capillary waves turbulence on water (Shats et al., 2010) and acoustic turbulence (Ganshin et al., 2008). They are characterised by long tails of their probability density functions (PDF) due to their very low probability of occurrence. The possibility to reproduce such rogue waves in optical temporal systems (Hammani et al., 2008; Solli et al., 2008) with characteristics similar to those encountered in the ocean is not surprising since the self-focusing Nonlinear Shrödinger Equation (NLSE) reflects the temporal nonlinear dynamics of waves propagating in both fibre optics (Agraval, 1995) and ocean surface (Za-

Correspondence to: E. Louvergneaux (eric.louvergneaux@univ-lille1.fr) kharov, 1968; Onorato et al., 2001; Kharif and Pelinovsky, 2003). These rogue events were also predicted and studied in spatially extended systems (Kim and Ott, 2003; Du et al., 2008) and discrete optical systems (Bludov et al., 2009) before being experimentally evidenced very lately in an optical ring cavity (Montina et al., 2009) and in laser filamentation (Kasparian et al., 2009). This leads to one of the main and attractive research activity in optics. The study of their formation in optical systems could provide, for example, inputs on mechanisms of their marine counterparts. Moreover, while the appearance of rogue waves in the ocean is a dreaded phenomenon, the formation and control of such pulses of very high energy in optics remains a challenge. Knowledge of the mechanisms of formation of these giant waves could, therefore, help to generate them deterministically rather than relying on random conditions.

Here, we report on the generation of rogue spatial peaks in the transverse patterns of a Kerr slice medium subject to optical feedback. These abnormal highly intensity peaks are observed in the turbulent regime (D'Alessandro and Firth, 1992) far above the primary instability threshold corresponding to the appearance of a stationary Turing pattern (corresponding to the well-known spatial modulational instability). This highly nonlinear regime in spatially extended optical systems that has not been characterised so far is numerically and experimentally studied in term of statistics and features of rogue spatial peaks. We show that their probability density functions are characterised by long tails indicating the presence of peaks that occur very rarely in comparison with the commonly time appearance frequency and with intensities far greater than those associated with typical peaks. We also emphasize that the highest amplitude peaks reach the criterion for rogue events (larger than 2 times the significant peak height) used in ocean waves (Onorato et al., 2001; Kharif and Pelinovsky, 2003).

Published by Copernicus Publications on behalf of the European Geosciences Union. 


\section{The experimental optical system}

The experimental setup in which optical spatial rogue peaks of intensity occur is composed of a Kerr slice medium subjected to optical feedback (Fig. 1) (Louvergneaux., 2001). This system configuration allows the generating of a rich variety of transverse patterns (from rolls or hexagons to spatial solitons) and has been extensively investigated, see, for example, (D'Alessandro and Firth, 1992; Denz et al., 1998; Arecchi et al., 2000; Ackemann and Lange, 2001; Agez et al., 2006). Here, it essentially consists of a nematic liquid crystal (LC) layer irradiated by a strong laser beam which is reflected back onto the sample by a plane mirror $M$ placed at a variable distance $d$ from the LC layer (Fig. 1). $R$ is the mirror intensity reflectivity. The nonlinear medium is a $50 \mu \mathrm{m}$ thick layer of $E_{7}$ LC homeotropically anchored. The beam is delivered by a monomode frequency doubled $\mathrm{Nd}^{3+}: \mathrm{YVO}_{4}$ laser $\left(\lambda_{0}=532 \mathrm{~nm}\right)$ which is shaped by means of two cylindrical lenses $C_{1}$ and $C_{2}$ in order to achieve a transverse quasi-monodimensional (1-D) pumping (beam diameters $\approx 240 \mu \mathrm{m} \times 3200 \mu \mathrm{m}$ ). The optical distance $d$ may be positive or negative with the help of an optical system made of two lenses in a $4 \mathrm{f}$-arrangement. Note that the equivalent medium is self-focusing (resp. defocusing) if $d>0$ (resp. $d<0$ ) (Ciaramella et al., 1993). The transmitted backward beam $B_{\text {out }}$ is monitored by CCD cameras. Near and far fields are simultaneously recorded. The two control parameters are the maximum intensity $I_{0}$ of the incident laser beam and the distance $d$ between the mirror and the LC layer. The reorientation of the LC director by the laser electric field leads to a nonlinear change of its refractive index $n$ which depicts pattern formation (Firth, 1990).

Typical 1-D transverse periodic pattern obtained at threshold for Turing instability is shown on Fig. 2a. It is the counterpart of modulational instability in temporal systems. Indeed, the optical Fourier transform of this near field pattern shows two spatial modes with critical wave numbers $k= \pm 2 \sqrt{\lambda d}$. The temporal recording of the near field profile along the long axis of the pattern (horizontal on Fig. 2) shows that the dynamics is stationary (Fig. 2c). Below this threshold, the transmitted field through the mirror $M$ simply reflects the overall Gaussian dependence of the laser input shape. On the other hand, as the input power is increased above the threshold, the periodic spots first destabilize via time pseudo-periodic dislocations associated with fringe creation in the spatiotemporal diagram (Louvergneaux., 2001), then a regime of turbulence is reached for input laser field values very far above threshold (Fig. 2d). The associated pattern (Fig. 2b) is composed of a very large number of transverse modes and its spatiotemporal dynamics is rather chaotic (D'Alessandro and Firth, 1992).

No analytical studies of this strongly nonlinear regime (far beyond threshold) have been carried out, since the dynamical description in terms of amplitude equations fails. Indeed, these kind of studies are performed near threshold where

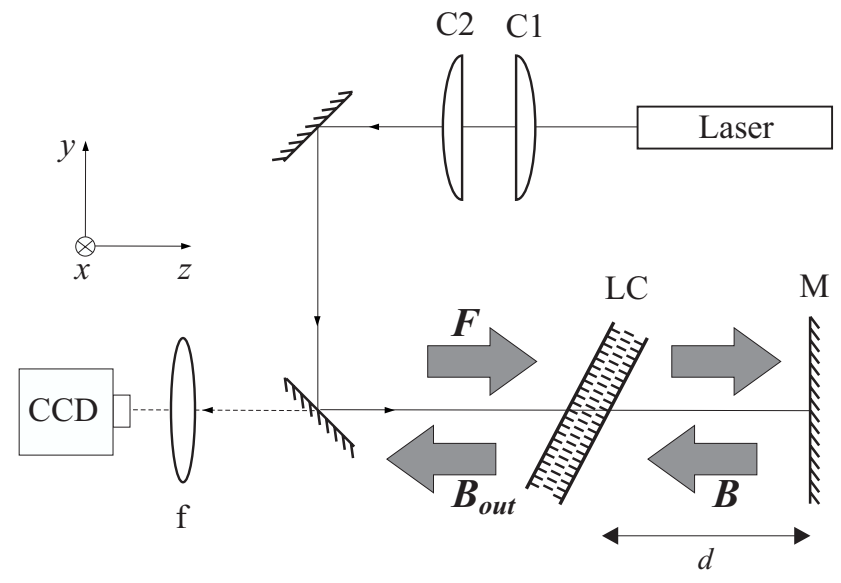

Fig. 1. Schematic sketch of the experimental set-up. LC liquid crystal layer; $M$ feedback mirror; $F$ input optical field; $B_{\text {out }}$ transmitted backward optical field; $B$ backward optical field; $d$ feedback length. $C_{1}$ and $C_{2}$ cylindrical lenses. $f$ near field imaging lens.

amplitude equations, mainly Ginzburg-Landau and SwiftHohenberg equations, can be derived to describe weakly nonlinear dynamics above threshold (Cross and Hohenberg, 1993). To our knowledge, except the transition to this spatiotemporal chaotic regime using time-average patterns and correlation functions (Neubecker et al., 1999), and a characterisation of two different states based on correlation functions and on Karhunen-Loeve decomposition (Pastur et al., 2003), no other characterisation of this turbulent regime has been done up to now. Here, we adopt a different statistical approach in terms of PDF of pattern maxima for the analysis of these highly nonlinear regimes. This evidence that rare and extreme peaks erratically appear in the pattern in the form of a very narrow spatial "pulse" with an amplitude far greater than the ones of the other intensity peaks of the transverse structure. In many aspects they are similar, and represent the spatial counterpart of what is known as rogue waves in hydrodynamics and very recently in optical temporal fibre systems.

\section{The model}

The modelisation of the previous experimental setup is well described in the framework of a model introduced first by Akhmanov et al. (1988) and later adapted by Firth and d'Alessandro (Firth, 1990). The equation for refractive index $n$ of the nonlinear nematic LC layer which captures the dynamics in the one-dimension case reads (D'Alessandro and Firth, 1992):

$\frac{\partial n}{\partial t}-\frac{\partial^{2} n}{\partial x^{2}}+n=|F|^{2}+|B|^{2}$

where $t, x$ are the time and transverse space variables scaled with respect to the relaxation time $\tau$ and the diffusion length 
(a)

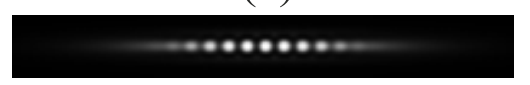

(c)

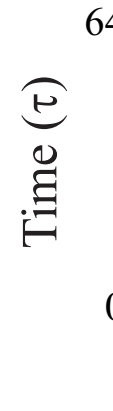

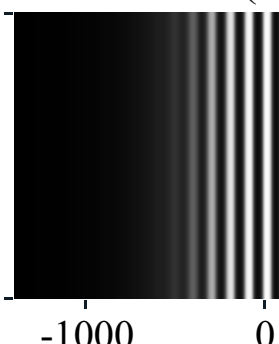

0

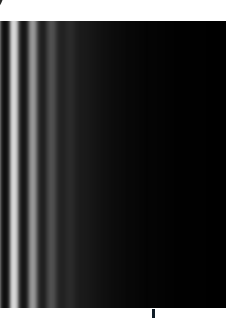

1000

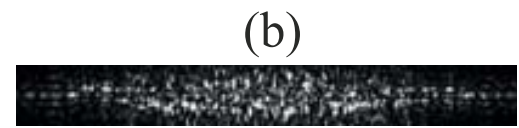

(d)

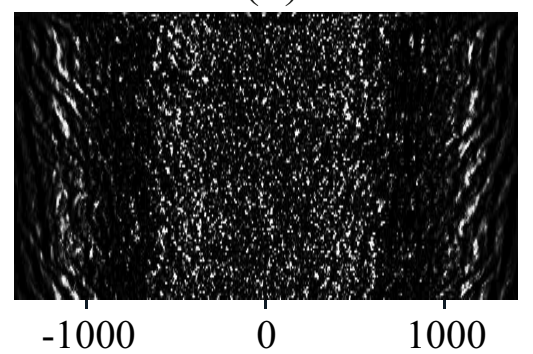

Transverse space $(\mu \mathrm{m})$

Fig. 2. Near field transverse patterns observed at (a) threshold and (b) in the turbulent regime. (c, d) Spatiotemporal diagrams of the pattern profile evolution taken along the horizontal axis and in the middle of the near field patterns (a) and (b), respectively. Transverse size of (a) and (b) pictures are $2800 \times 350 \mu \mathrm{m}$. The numerical simulations have been performed following the model of Eqs. (1-3) for a Gaussian input wave pumping. $w=1400 \mu \mathrm{m}, \chi=1, d=5 \mathrm{~mm}, R=0.9$. (a, c) $F_{0}=0.92$ and (b, d) $F_{0}=4.5$.

$l_{D}$, respectively. The diffusion length $l_{D}$ inside the crystal is much larger than the optical wavelength $\lambda_{0}$, thus, removing the longitudinal interference effects. The small thickness of the Kerr medium allows neglecting light diffraction along the sample (Firth, 1990). $F$ and $B$ in Eq. (1) are the slowly varying amplitudes of the forward and backward fields inside the liquid crystal sample, respectively (Fig. 1). $B$ is obtained using the two following equations that govern the propagation of $F$ along the $z$ optical axis over the feedback loop. Equation (2) accounts for the phase modulation through the LC sample and Eq. (3) for the propagation through the free space (D’Alessandro and Firth, 1992):

$\frac{\partial F}{\partial z}=i \chi n F$

$\frac{\partial F}{\partial z}=\frac{i}{2 k_{0}} \frac{\partial^{2} F}{\partial x^{2}}$.

$\chi$ parameterizes the Kerr effect (positive for a focusing medium, $d>0$ ) and $k_{0}$ is the laser field wave number. The profile of the forward field at the entrance of the liquid crystal is assumed to be Gaussian so that $F(x)=F_{0} \exp \left(-x^{2} / w^{2}\right)$, with $w$ the beam radius at sample.

Note that the quantity we can experimentally access is the backward output optical beam intensity that we have set $I=$ $\left|B_{\text {out }}\right|^{2}$ (see Fig. 1). This is our dynamical variable that is monitored on the near field imaging CCD camera and that is used for the study of the probability density functions. It reads:

$\left|B_{\text {out }}(x, t)\right|^{2}=\left|e^{i \chi n(x, t)} B(x, t)\right|^{2}$

\section{Numerical rogue patterns}

The numerical simulations carried out in the very nonlinear regime typically depict turbulent spatiotemporal dynamics of the near field $B_{\text {out }}$ as can be seen on Fig. $2 d$. The spatiotemporal diagram is complex but a deeper look on the evolution of the transverse profile shows the existence of very intense spatial peaks as the one observed on Fig. 3a. It corresponds to a temporal snapshot of the transverse cross-section of the 1-D pattern (Fig. 2b) and representing a horizontal line of the corresponding spatiotemporal diagram in Fig. 2d. Such an intense peak is many times the intensity of the other surrounding peaks. To characterise these peaks, we proceed by a statistical approach using the probability density function (PDF) of the intensity of pattern peaks. We calculate the PDFs as the histograms of the intensity maxima along the 1-D spatial pattern for a given recording time (few hundreds of relaxation time $\tau$ ). The PDFs must be plotted for uniformlike pumping conditions. So, we performed all our PDF calculations on a restricted spatial zone of the Gaussian input pump. This area is located in the central part and its extension must be smaller than $0.5 w$ (with $2 w$ is the beam diameter) to keep the PDFs independent of the Gaussian shape.

A typical PDF profile associated with the turbulent regime is depicted in Fig 3b. It possesses a long tail that is characteristic of very strong events such as the one displayed in red in Fig 3a. To characterise these events as freak events, we use the criterion developed for the oceanic rogue waves and which states that their height must exceed 2 times the significant wave height $H_{S}$ (Kharif and Pelinovsky, 2003). In our system, the counterpart of the oceanic wave height is the optical near field peak amplitude $B_{\text {out }}$. Here the significant amplitude is $B_{\text {out }, s}=7.09$. The highest amplitude value 

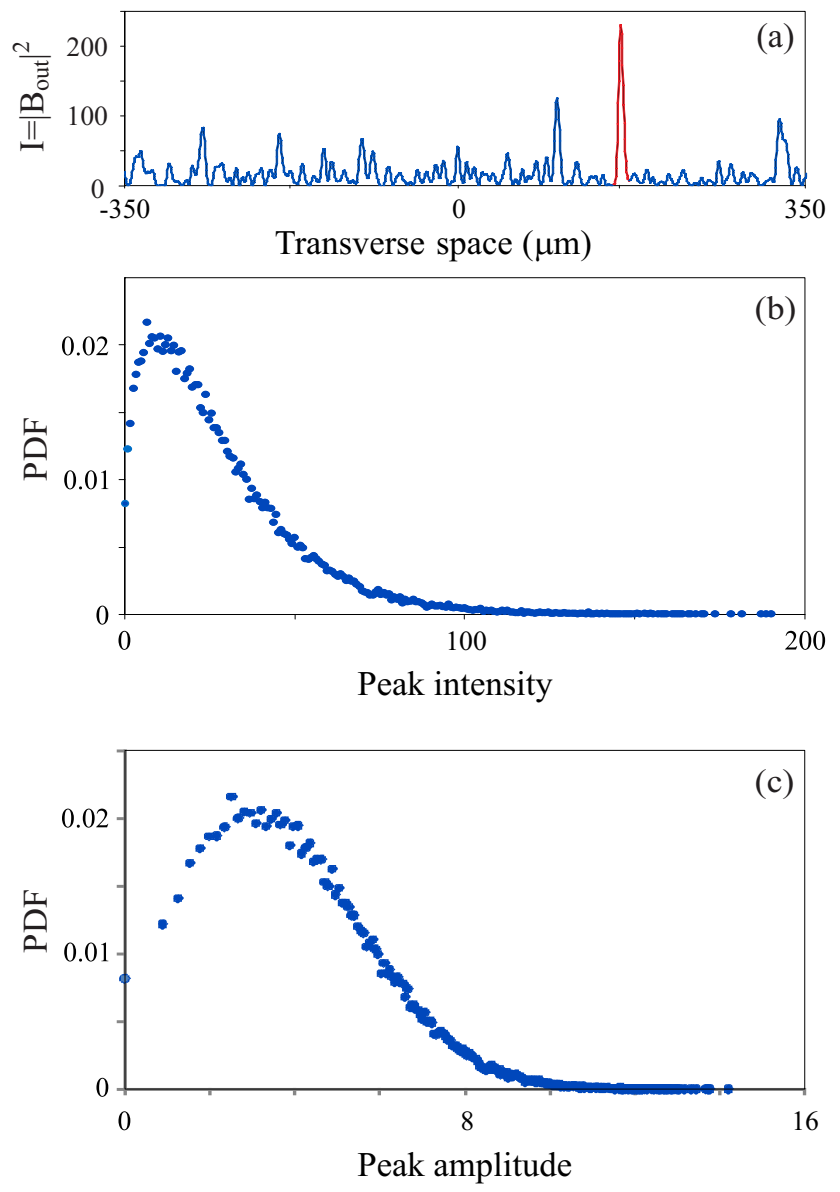

Fig. 3. (a) Transverse cross-section of 1-D pattern of Fig. 2b taken along the horizontal axis showing a typical spatial rogue event (red peak). (b) Associated numerical probability density function (PDF) versus peak intensity. (c) PDF versus peak amplitude. The numerical simulations have been done following the model of Eq. (1) for a Gaussian input wave pumping. $w=1400 \mu \mathrm{m}, \chi=1, d=5 \mathrm{~mm}$, $F_{0}=4.5, R=0.9$.

slightly exceeds 2 times $B_{\text {out,s }}$ (Fig. 3c). The percentage of peaks of this PDF that have an amplitude 2 times larger than that of the significant peak amplitude is about $0.8 \%$. Thus, all of them can be classified as rogue peaks.

Another characteristic of rogue events is that they appear and disappear in a very "short" time. Here, their lifetime are of the order of the relaxation time $\tau$ of the system, namely, less than 0.5 times $\tau$ which is the temporal resolution of our numerical simulations. Thus, the very intense spatial peaks of our system "appear from nowhere and disappear without a trace" which is one of the established signatures of the rogue waves in both the ocean and in the fibre supercontinuum generation (Akhmediev et al., 2009).
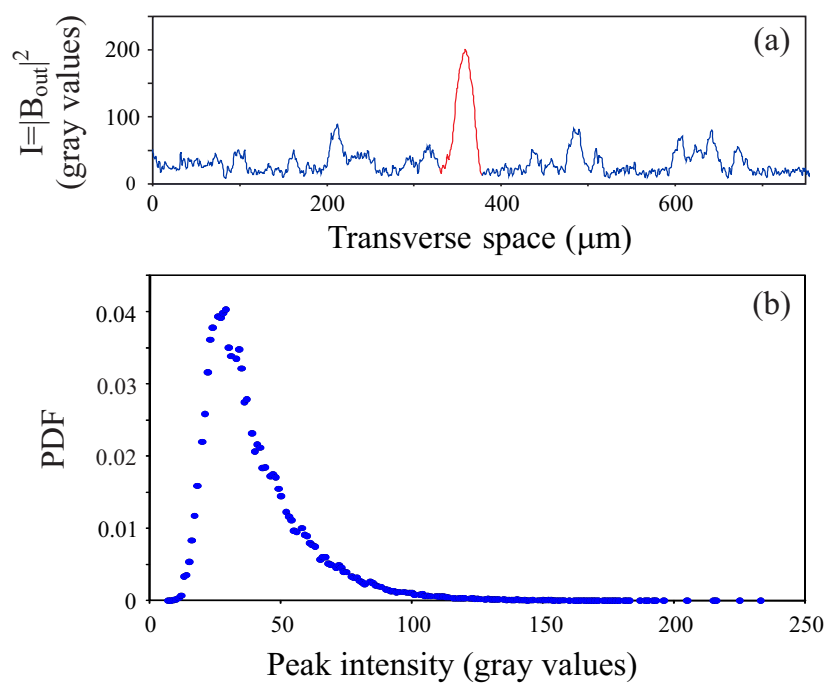

Fig. 4. (a) Transverse cross-section of a 1-D experimental pattern taken along the horizontal axis showing a typical experimental spatial rogue event (red peak). (b) Associated experimental probability density function (PDF) versus peak intensity. $d=5 \mathrm{~mm}$, $w=1600 \mu \mathrm{m}$. Input intensity is 6 times intensity threshold for modulational instability.

\section{Experimental evidence of rogue patterns}

The experimental study of the spatiotemporal chaotic regime is carried out in an optical system pumped by a transverse quasi-one-dimensional (1-D) laser beam. As was mentioned in the experimental setup description, only one roll is allowed in the vertical transverse direction. In these conditions, a line of spots similar to the one of Fig. 2a rises up at threshold of the modulational instability. Further increasing the laser pump power, the regime of spatial turbulence is reached. However, the liquid crystal slice can not be approximated to a Kerr-like system for very high pump powers where spatial self-focusing starts (for typically pump powers larger than 6 to 8 times the threshold for modulation instability). The 1-D pattern then expands in the transverse orthogonal direction to the 1-D horizontal axis (Khoo, 1995) and can no more be considered as a 1-D pattern. Thus, a limitation on the maximum input pump intensity value exists restricting the spatiotemporal chaotic regime domain that can be studied experimentally. Even under these restrictions it is still sufficient to experimentally evidence the regime of spatial rogue events.

The near field intensity patterns (i.e., within the liquid crystal cell) are recorded with an 8 bits resolution depth CCD camera (Fig. 1). At modulational instability threshold the transverse wavelength is around $100 \mu \mathrm{m}$ so that the aspect ratio is around 30. At 6 times this intensity threshold, very intense localized spatial peaks, such as the red one on Fig. 4a, rarely emerge and disappear as fast as they appeared. The corresponding typical PDF is plotted on Fig. 4b. Its shape 
is completely similar to the one predicted in Fig. $3 \mathrm{~b}$ and is a signature of spatial rogue peaks in the 1-D transverse patterns. Only a shift is observed on the horizontal axis due to the presence of the CCD zero intensity background. As for the numerical simulations, the experimental highest amplitude value (Fig. 4b) exceeds 2 times (2.1) the significant peak amplitude $B_{\text {out }, s}=7.14$, so that the criterion for rogue event is fulfilled. Thus, the occurrence of rogue spatial peaks in the turbulent regime of 1-D transverse patterns is experimentally evidenced.

\section{Conclusions}

Using a statistical approach, we have numerically and experimentally evidenced the generation of rogue spatial peaks in the one-dimensional transverse patterns of a liquid crystal slice subject to optical feedback. The regime in which they occur is highly nonlinear (far above the threshold for modulational instability also known as Turing instability) and corresponds to a turbulent spatiotemporal dynamics. The probability density function shows the very rare nature of these events. In addition, the spatiotemporal diagrams of the 1-D patterns demonstrate their very short lifetime. More importantly, the spatial rogue patterns evidenced here are similar to many aspects to the rogue waves encountered in open ocean and supercontinuum generation in photonic crystal fibres.

\section{Edited by: E. Pelinovsky}

Reviewed by: V. Konotop and another anonymous referee

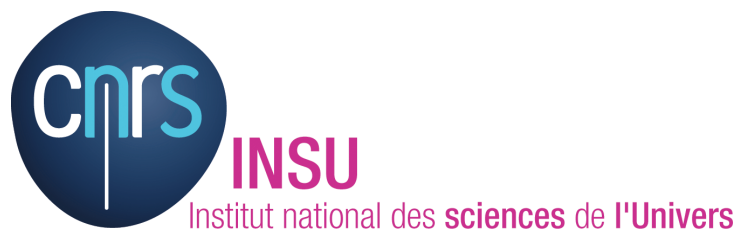

The publication of this article is financed by CNRS-INSU.

\section{References}

Ackemann, T. and Lange, W.: Optical pattern formation in alkali metal vapors: Mechanism, phenomena and use, Appl. Phys. B, 72, 21-34, 2001.

Agez, G., Glorieux, P., Taki, M., and Louvergneaux, E.: Twodimensional noise-sustained structures in optics: Theory and experiments, Phys. Rev. A, 74, 043814, 2006.

Agraval, G. P.: Nonlinear fibre optics, Academic Press, San Diego, California, 26 pp., 1995.

Akhmanov, S., Vorontsov, M., and Ivanov, V. Y.: Large-scale transverse nonlinear interactions in laser beams; new types of nonlinear waves; onset of optical turbulence, JETP Lett., 47, 707, 1988.
Akhmediev, N., Ankiewicz, A., and Taki, M.: Waves that appear from nowhere and disappear without a trace, Phys. Lett. A, 373, 675-678, doi:10.1016/j.physleta.2008.12.036, http: //www.sciencedirect.com/science/article/B6TVM-4V74XJY-3/ 2/537aefb6736ea6c45fc2fa3a2420c39d, 2009.

Arecchi, F., Boccaletti, S., Ducci, S., Pampaloni, E., Ramazza, P., and Residori, S.: The liquid crystal light valve with optical feedback: a case study in pattern formation, J. Nonlinear Opt. Phys., 9, 183, 2000

Bludov, Y. V., Konotop, V. V., and Akhmediev, N.: Rogue waves as spatial energy concentrators in arrays of nonlinear waveguides, Opt. Lett., 34, 3015-3017, doi:10.1364/OL.34.003015, http://ol. osa.org/abstract.cfm?URI=ol-34-19-3015, 2009.

Broad, W.: Rogue giants at sea, The New York Times, http://www. nytimes.com/2006/07/11/science/11wave.html, July 112006

Ciaramella, E., Tamburini, M., and Santamato, E.: Talbot assisted hexagonal beam pattening in a thin LC film with a single feedback miror at negative distance, Appl. Phys. Lett., 63, 1604, 1993.

Cross, M. C. and Hohenberg, P. C.: Pattern formation outside equilibrium, Rev. Mod. Phys., 65, 851, 1993.

D'Alessandro, G. and Firth, W. J.: Hexagonal spatial patterns for a Kerr slice with a feedback mirror, Phys. Rev. A, 46, 537-548, 1992.

Denz, C., Schwab, M., Sedlatschek, M., Tschudi, T., and Honda, T.: Pattern dynamics and competition in a photorefractive feedback system, J. Opt. Soc. Am. B, 15, 2057-2064, http://josab.osa.org/ abstract.cfm?URI=josab-15-7-2057, 1998.

Du, L., Chen, Q., Lai, Y.-C., and Xu, W.: Observation-based control of rare intense events in the complex Ginzburg-Landau equation, Phys. Rev. E, 78, R015201, doi:10.1103/PhysRevE.78.015201, 2008.

Dudley, J., Genty, G., and Eggelton, B.: Harnessing and control of optical rogue waves in supercontinuum generation, Opt. Express, 16, 3644-3651, 2008

Fedele, F.: Rogue waves in oceanic turbulence, Physica D: Nonlinear Phenomena, 237, 2127-2131, doi:10.1016/j.physd.2008.01. 022, euler Equations: 250 Years On - Proceedings of an international conference, 2008.

Firth, W. J.: Spatial instabilities in a Kerr medium with single feedback mirror, J. Mod. Opt., 37, 151-153, 1990.

Ganshin, A. N., Efimov, V. B., Kolmakov, G. V., MezhovDeglin, L. P., and McClintock, P. V. E.: Observation of an Inverse Energy Cascade in Developed Acoustic Turbulence in Superfluid Helium, Phys. Rev. Lett., 101, 065303, doi:10.1103/PhysRevLett.101.065303, 2008.

Hammani, K., Finot, C., Dudley, J. M., and Millot, G.: Optical rogue-wave-like extreme value fluctuations in fibre Raman amplifiers, Opt. Express, 16, 16467-16474, 2008.

Kasparian, J., Béjot, P., Wolf, J., and Dudley, J.: Optical rogue wave statistics in laser filamentation, Opt. Express, 17, 12070-12075, 2009.

Kharif, C. and Pelinovsky, E.: Physical mechanisms of the rogue wave phenomenon, Eur. J. Mech. B/Fluids, 22, 603-634, 2003.

Khoo, I.: Liquid crystals : physical properties and nonlinear optical phenomena, John Wiley \& Sons, 225 pp., 1995.

Kim, J.-W. and Ott, E.: Statistics and characteristics of spatiotemporally rare intense events in complex Ginzburg-Landau models, Phys. Rev. E, 67, 026203, doi:10.1103/PhysRevE.67.026203, 
2003.

Louvergneaux, E.: Pattern dislocation-type dynamical instability in 1-D optical feedback Kerr media with gaussian transverse pumping, Phys. Rev. Lett., 87, 244501, doi:10.1103/PhysRevLett.87.244501, 2001.

Montina, A., Bortolozzo, U., Residori, S., and Arecchi, F. T.: Non-Gaussian Statistics and ExtremeWaves in a Nonlinear Optical Cavity, Phys. Rev. Lett., 103, 173901, doi:10.1103/PhysRevLett.103.173901, 2009.

Mussot, A., Kudlinski, A., Kolobov, M., Louvergneaux, E., Douay, M., and Taki, M.: Observation of extreme temporal events in CW-pumped supercontinuum, Opt. Exp., 17, 17010, doi:10.1364/OE.17.017010, 2009.

Neubecker, R., Thuring, B., Kreuzer, M., and Tschudi, T.: Transition from Spatio-temporal Order to Disorder in a Single-feedback Experiment, Chaos, Solitons \& Fractals, 10, 681-692, doi:10.1016/S0960-0779(98)00016-2, http: //www.sciencedirect.com/science/article/B6TJ4-41DJHY2-8/2/ aa02cad8d6a35dcafc75b506ba992c1b, 1999.

Onorato, M., Osborne, A. R., Serio, M., and Bertone, S.: Freak Waves in Random Oceanic Sea States, Phys. Rev. Lett., 86, 5831-5834, doi:10.1103/PhysRevLett.86.5831, 2001.
Pastur, L., Bortolozzo, U., and Ramazza, P.: Experimental characterization of space-time chaos in nonlinear optics, in: EXPERIMENTAL CHAOS, edited by: In, V, Kocarev, L., and Carroll, T. L., Gluckman, B. J., Boccaletti, S., and Kurths, J., 676 of AIP CONFERENCE PROCEEDINGS, 182-188, 7th Experimental Chaos Conference, SAN DIEGO, CA, 26-29 August 2002, 2003.

Shats, M., Punzmann, H., and Xia, H.: Capillary Rogue Waves, Phys. Rev. Lett., 104, 104503, doi:10.1103/PhysRevLett.104. 104503, 2010.

Solli, D. R., Ropers, C., Koonath, P., and Jalali, B.: Optical rogue waves, Nature, 450, 1054-1058, 2007.

Solli, D. R., Ropers, C., and Jalali, B.: Active Control of Rogue Waves for Stimulated Supercontinuum Generation, Phys. Rev. Lett., 101, 233902, doi:10.1103/PhysRevLett.101.233902, http: //link.aps.org/abstract/PRL/v101/e233902, 2008.

Zakharov, V. E.: Stability of periodic waves of finite amplitude on the surface of a deep fluid, J. Appl. Mech. Tech. Phys., 9, 190-194, doi:10.1007/BF00913182, http://www.springerlink. com/content/h0162g222573r988, 1968. 